\title{
Broadband Shifts in Local Field Potential Power Spectra Are Correlated with Single-Neuron Spiking in Humans
}

\author{
Jeremy R. Manning, ${ }^{1}$ Joshua Jacobs, ${ }^{2}$ Itzhak Fried, ${ }^{3,4,5,6}$ and Michael J. Kahana ${ }^{2}$ \\ ${ }^{1}$ Neuroscience Graduate Group and ${ }^{2}$ Department of Psychology, University of Pennsylvania, Philadelphia, Pennsylvania 19104, ${ }^{3}$ Division of Neurosurgery \\ and ${ }^{4}$ Semel Institute of Neuroscience and Human Behavior, University of California, Los Angeles, California 90095, and ${ }^{5}$ Functional Neurosurgery Unit, \\ Tel-Aviv Medical Center, and ${ }^{6}$ Sackler Faculty of Medicine, Tel-Aviv University, Tel-Aviv 69978, Israel
}

A fundamental question in neuroscience concerns the relation between the spiking of individual neurons and the aggregate electrical activity of neuronal ensembles as seen in local field potentials (LFPs). Because LFPs reflect both spiking activity and subthreshold events, this question is not simply one of data aggregation. Recording from 20 neurosurgical patients, we directly examined the relation between LFPs and neuronal spiking. Examining 2030 neurons in widespread brain regions, we found that firing rates were positively correlated with broadband $(2-150 \mathrm{~Hz})$ shifts in the LFP power spectrum. In contrast, narrowband oscillations correlated both positively and negatively with firing rates at different recording sites. Broadband power shifts were a more reliable predictor of neuronal spiking than narrowband power shifts. These findings suggest that broadband LFP power provides valuable information concerning neuronal activity beyond that contained in narrowband oscillations.

\section{Introduction}

A large and growing literature has documented the existence of oscillatory activity from large neuronal ensembles in both humans and animals, and has shown that these brain oscillations are related to a wide variety of behavioral and cognitive states (Kahana et al., 2001; Buzsáki, 2004, 2006; Crone et al., 2006; Jacobs and Kahana, 2009; Jerbi et al., 2009). Elucidating the relation between large-scale neural patterns and the activities of individual neurons is critical for gaining a deep understanding of the brain and how it supports behavior (Kreiman, 2007). To this end, several studies of humans and animals have examined the relation between large-scale recordings, such as local field potentials (LFPs), and the activities of individual neurons.

Recording from monkey visual cortex, Rasch et al. (2008) found that increases in gamma activity $(40-90 \mathrm{~Hz})$ and decreases in delta and theta activity $(1-10 \mathrm{~Hz})$ in the LFP signal were correlated with increased neuronal firing rates. Examining primary auditory cortex as human neurosurgical patients viewed brief movies, Mukamel et al. (2005) also observed that increased gamma activity and decreased theta activity correlated with neuronal firing. The positive correlation between gamma activity and neuronal spiking is a robust finding reported in a variety of studies (Fries et al., 2001; Pesaran et al., 2002).

\footnotetext{
Received April 21, 2009; revised Sept. 24, 2009; accepted Sept. 28, 2009.

We are grateful for the support of National Institutes of Health Grants MH61975, MH062196, T32 NS054575, NS033221, and F32 NS50067-01A1; National Science Foundation Grant SBE0354378; and Dana Foundation Grant Intracranial EEG for Theta Rhythm Contingency During Cognitive Tasks. We thank S. Polyn and C. Weidemann for valuable feedback on an earlier draft of this manuscript, A. Ekstrom for useful discussion on our approach, and L. Lohnas for help with calculations.

Correspondence should be addressed to Michael J. Kahana, Department of Psychology, University of Pennsylvania, 3401 Walnut Street, Room 303C, Philadelphia, PA 19104. E-mail: kahana@psych.upenn.edu.

D0I:10.1523/JNEUROSCI.2041-09.2009

Copyright $\odot 2009$ Society for Neuroscience $\quad$ 0270-6474/09/2913613-08\$15.00/0
}

Reports of strong correlations between neuronal firing and narrowband activity (i.e., oscillations) have supported the view that oscillations reflect synchronized spike timing in large neuronal ensembles (Singer and Gray, 1995; Logothetis, 2003; Fries et al., 2007). This follows in part from the temporal-binding hypothesis (von der Malsburg, 1981), which proposes that synchronized neural activity can solve the "binding problem" by linking multiple neuronal signals (Koffka, 1935; Köhler, 1947; Kanisza, 1979; Pal and Pal, 1993). However, several studies suggest that apparent correlations between spikes and gamma-band LFP activity are actually due to broadband LFP patterns, rather than band-specific oscillations (Mitzdorf, 1985; Juergens et al., 1999; Cruikshank et al., 2002; Kaur et al., 2004; Kreiman et al., 2006; Chen et al., 2007; Miller et al., 2007b). Furthermore, task-related modulations in human LFP activity have been observed throughout a broad frequency range extending to $\sim 150-200 \mathrm{~Hz}$, showing that the human brain exhibits broadband phenomena in addition to narrowband oscillations (Lachaux et al., 2005; Tanji et al., 2005; Canolty et al., 2006). Other work suggests that broadband changes in LFP activity are related to neuronal spiking (Miller et al., 2009; Milstein et al., 2009). However, no previous work has directly compared neuronal spiking in humans with simultaneous broadband LFP activity.

Here we examined the relation between LFPs and singleneuron activity in 20 neurosurgical patients during a virtualnavigation task. We recorded action potentials from 2030 neurons from diverse brain regions including the neocortex and medial temporal lobe. Consistent with previous studies, we found a population of narrowband-shift neurons, which varied their firing in proportion to LFP power at specific frequency bands. Narrowband-shift neurons were present throughout the brain, but were especially prevalent in the frontal cortex and amygdala. In addition, we observed a larger population of broad- 
band-shift neurons, which varied their firing with the overall height of the LFP power spectrum at all frequencies. Broadbandshift neurons appeared in all examined brain regions, but were especially prevalent in the medial temporal lobe. Broadband increases in LFP power were almost exclusively positively correlated with single-neuron firing, providing a robust estimate of neuronal firing. We propose that, when estimating local neuronal firing using LFPs, researchers should examine broadband power in addition to power contained in narrow frequency bands.

\section{Materials and Methods}

Electrophysiological recordings. We examined intracranial brain recordings from 20 neurosurgical patients undergoing treatment for drugresistant epilepsy. During each 25-60 min recording session, patients played a virtual-navigation game, Yellow Cab, in which they assume the role of a taxi driver and chauffeur (virtual) passengers to their desired destinations. While playing this game, patients learn the virtual environment's layout (Newman et al., 2007) and display brain oscillations at various frequencies related to learning and sensorimotor integration (Caplan et al., 2003; Ekstrom et al., 2005).

Patients are implanted with 6-12 neurosurgical depth electrodes by clinical teams. LFPs recorded from these electrodes are used to map the seizure focus and identify functional brain regions for potential subsequent surgical resection. In addition, extending from the tip of each depth electrode is a set of nine small microwires. (The ninth wire is the recording reference for the other eight.) Each microwire is $40 \mu \mathrm{m}$ in diameter and records from a small, local region of cortex. By recording from the microwires at $32 \mathrm{kHz}$, we observe both high-frequency singleneuron spiking (Fried et al., 1999) and lower-frequency LFPs (Mukamel et al., 2005; Jacobs et al., 2007). Using the WaveClus software package (Quiroga et al., 2004), we identified the action potentials of $0-3$ neurons per microwire, for a total of 2030 neurons across 20 subjects. Recordings were obtained in widespread brain regions including the frontal cortex, posterior cortex (occipital and parietal cortices), amygdala, hippocampus, and parahippocampal region (Witter, 2002). To make the LFP data more computationally tractable, we downsampled our recordings to 2 $\mathrm{kHz}$. We then applied a second-order Butterworth notch filter at $60 \mathrm{~Hz}$ to remove line noise. We computed the smoothed firing rate for each time point by convolving each neuron's spike train with a Gaussian kernel (half-width $=500 \mathrm{~ms}$ ). To prevent the low-frequency components of the action-potential waveform from contaminating the LFP signal, we replaced the data samples in the -2 to $8 \mathrm{~ms}$ window around each spike with a linear interpolation of the underlying LFP signal (Jacobs et al., 2007). These recordings were obtained for a previous study (Jacobs et al., 2007), but the analyses reported here are completely novel.

LFP feature extraction. We measured oscillatory power in the LFP signal using Morlet wavelets (wave number $=4$ ) at $50 \log$-spaced frequencies between 2 and $150 \mathrm{~Hz}\left(2 \times 10^{0.0383 x}\right.$ for $\left.x \in\{0 \ldots 49\}\right)$. Because oscillatory power at a given frequency is $\chi^{2}$ distributed (Percival and Walden, 1993; Henrie and Shapley, 2005), we log-transformed the wavelet-calculated powers to make the distributions more normal. To account for interelectrode impedance differences, we normalized the powers recorded at each electrode such that the mean power spectrum was centered at 0 with an SD of 1 .

To analyze the relation between LFP spectral power and spiking activity, we next divided each recording session into $500 \mathrm{~ms}$ epochs. This epoch length was chosen to provide a reasonable balance between temporal resolution (which we sought to maximize) and correlations across successive measurements (which we sought to minimize). To eliminate the effect of nonbiological noise on our analysis, we removed epochs with firing rates above the 99th percentile. For computing summary statistics, we calculated the mean power contained in the following narrow frequency bands: delta $(2-4 \mathrm{~Hz})$, theta $(4-8 \mathrm{~Hz})$, alpha $(8-12 \mathrm{~Hz})$, beta $(12-30 \mathrm{~Hz})$, and gamma $(30-150 \mathrm{~Hz})$. In addition to measuring LFP power at narrow frequency bands, we computed broadband power. Broadband power refers to LFP voltage fluctuations that occur at a broad range of frequencies, consistent with the voltage following a randomwalk process (Annibaldi and Hopcraft, 2002), unlike true oscillations limited to a narrow frequency range. To measure the broadband power for each individual epoch, we fit a line to that epoch's wavelet-calculated power spectrum via a robust regression (Holland and Welsch, 1977). Robust regression fits the majority of data points closely and is relatively unaffected by outliers; in contrast, a standard least-squares regression would be more affected by data points that did not fall on the line. This is critical for distinguishing broadband and narrowband effects because it minimizes the impact of narrowband oscillations, which appear as local peaks in the LFP power spectrum, toward the computed broadband power. We use the term "broadband power" to refer to the mean height of the robust-regression-fitted line in each epoch.

Regression framework. Our primary objective was to examine how the firing rates of individual neurons related to narrowband changes (i.e., oscillations) and broadband changes in the LFP. To quantify the extent to which each of these patterns predicted neuronal firing, we used a series of least-squares regressions. For each neuron, we set up five bivariate simultaneous regressions of the form $R=\beta_{0}+\beta_{B} B+\beta_{F} F$, where $R$ was a vector containing the estimated firing rate for each epoch, $\boldsymbol{F}$ was a vector containing the average power in each epoch for one narrowband frequency (delta, theta, alpha, beta, or gamma), and $\boldsymbol{B}$ was a vector containing broadband power. Before computing the mean power in each narrow frequency band for each electrode, we $z$-transformed the power distribution at each individual frequency to have a mean of 0 and an SD of 1 . This step ensured that the individual frequencies in each band contributed equally, despite the overall $1 / f^{a}$ shape of the power spectrum. The regression was performed five times (once for each frequency band), and the regression coefficients, $\beta_{0}, \beta_{B}$, and $\beta_{F}$, were fit separately each time. When the $\beta_{B}$ coefficients from all five regressions were significantly different from zero (see bootstrap procedure, below), and all had the same sign, we designated the neuron as a broadband-shift neuron. This technique ensured that, for broadband-shift neurons, broadband power explained a significant portion of the firing-rate variance beyond any one narrow frequency band alone.

We designated a neuron as a narrowband-shift neuron if exactly one $\beta_{F}$ was significantly different from zero (see bootstrap procedure, below). In addition, a neuron was also labeled as a narrowband-shift neuron if it had exactly two significant $\beta_{F}$ values with the same sign in neighboring frequency bands (e.g., $\beta_{\boldsymbol{\delta}}<0$ and $\beta_{\boldsymbol{\theta}}<0$ ). We added this second condition so that our analysis was not biased against neurons whose firing rates were related to oscillations that spanned the frequencyband boundaries. In this way, the firing rates of narrowband-shift neurons were correlated with oscillations in one narrow frequency band. Note that a single neuron could be tagged as both a broadband-shift neuron and a narrowband-shift neuron. As a potential alternative to the bivariate regression framework presented here, we also considered the use of a single simultaneous regression for each neuron (using broadband power and each of the five frequency bands as regressors). However, because the different frequency bands were highly colinear, results using this alternative approach were difficult to interpret.

Multiple comparisons. Our framework for classifying neurons as broadband- and narrowband-shift neurons relied on the outcomes of several potentially correlated statistical tests. To address this issue we used a bootstrap procedure to calculate a $p$ threshold to use for each statistic. We computed this threshold in a way that allowed us to set a single false-positive rate for the entire procedure (i.e., across all five regressions). We used a time-shifting bootstrap to estimate the probability of falsely labeling each neuron as a narrowband-shift neuron and as a broadband-shift neuron. For each neuron, we generated 1000 simulated firing rate vectors by circularly shifting the values of the original vector by a random number of elements. For each time-shifted vector, we re-ran the original regression analyses providing, for each neuron, 1000 sets of bootstrap regression coefficients and $p$ values. We used these bootstraps to determine, for each neuron, two $p$ thresholds-one for identifying narrowband-shift neurons and one for identifying broadband-shift neurons-that gave a 5\% false-positive rate for each designation. Although individual LFP recordings may exhibit differing correlations between the powers at neighboring frequencies, our procedure ensured that each neuron's false-positive rate is fixed at $5 \%$ for each effect. 

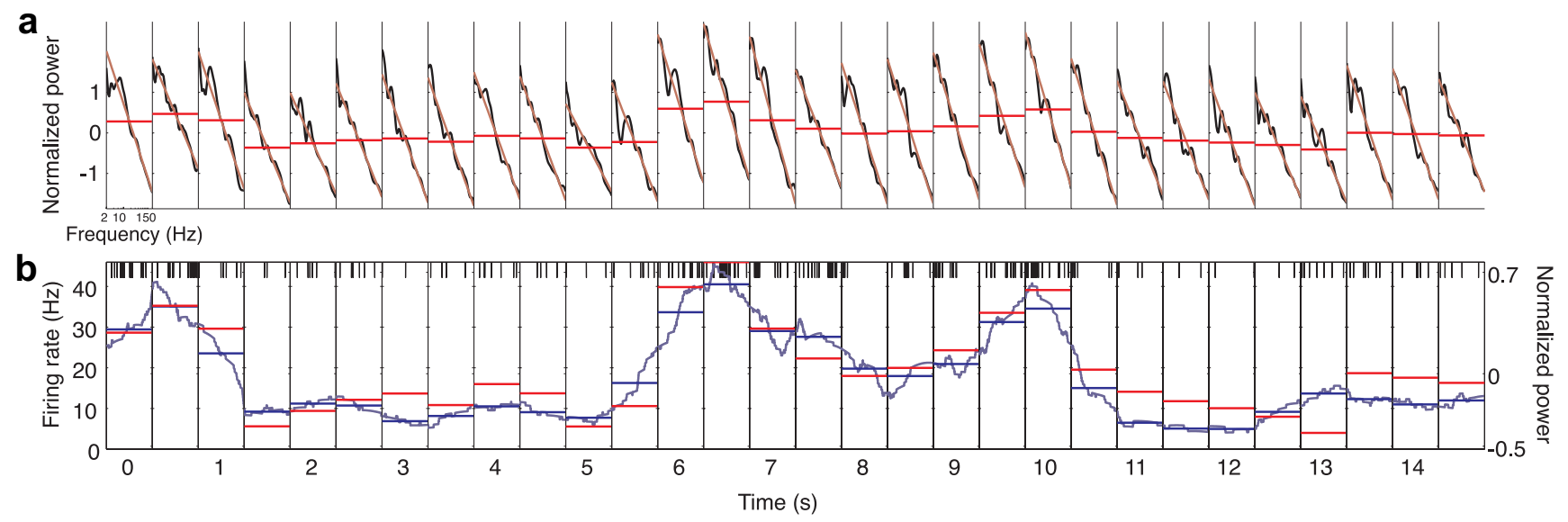

Figure 1. LFP power and neuronal firing time series. Each box details the activity in one $500 \mathrm{~ms}$ epoch. $\boldsymbol{a}$, This panel illustrates how various features of the LFP change over time. In each epoch, the black lines indicate the overall LFP power spectrum, brown lines indicate robust-fit lines, and the horizontal red lines indicate mean broadband powers. $\boldsymbol{b}$, This panel illustrates changes in neuronal firing rate concurrent with changes in the LFP power spectrum. Black vertical ticks represent the times when individual spikes occurred, dark blue lines indicates the smoothed firing rate (see Materials and Methods), and horizontal blue lines indicate mean firing rates in each epoch. Mean broadband power is shown in $\boldsymbol{b}$ (horizontal red lines) on a different scale (indicated at right).

\section{Results}

Using recordings of 2030 neurons from 20 neurosurgical patients (supplemental Table S1, available at www.jneurosci.org as supplemental material), we analyzed the relation between the firing rates of these neurons and simultaneous variations in the LFP. In particular, we determined how moment-to-moment variations in two distinct aspects of LFP power related to simultaneous changes in the firing rates of nearby neurons (supplemental Fig. S1, available at www.jneurosci.org as supplemental material). In addition to examining LFP oscillations in narrow frequency ranges, as traditionally done in this type of work (Kahana, 2006; Fries et al., 2007), we also analyzed a novel measure of broadband LFP activity. Broadband activity refers to changes in the LFP power spectrum that simultaneously appear at all frequencies, rather than being limited to a narrow frequency band. We measured broadband power using a technique that measures the overall level of the LFP power spectrum while ignoring narrowband oscillatory peaks (see Materials and Methods). This procedure ensures that whenever we observed a neuron whose spiking was correlated with broadband power, this activity was truly related to the overall level of the LFP power spectrum, rather than to oscillations at specific bands. We ruled out that broadband LFP power shifts are a consequence of low-frequency components of the spike waveform appearing in the LFP (see supplemental material, available at www.jneurosci.org). Before presenting aggregate statistics for the full dataset, we provide some sample data to illustrate the major phenomena of interest.

Figure 1 illustrates the relation between broadband power shifts and spiking activity recorded from an electrode in the amygdala of Patient 3. Figure $1 a$ shows the normalized LFP power spectrum (black lines) and the mean broadband LFP power (red lines) for each of 30 consecutive $500 \mathrm{~ms}$ epochs. Figure $1 b$ shows the neuron's spiking (black tick marks) and mean firing rate (blue lines) for these same epochs. Across these epochs, variations in broadband power were strongly correlated with simultaneous variations in the neuron's firing rate (Pearson's $r=$ $\left.0.92, p<10^{-12}\right)$. Note that both broadband power and neuronal firing rate exhibited local maxima at $0.5,4,6.5$, and $10 \mathrm{~s}$, and both had local minima at 1.5 and 5 s. Critically, variations in LFP power were not limited to particular narrow frequency bands, but rather appeared as overall broadband shifts in the entire power spectrum (Fig. 1a, brown lines).
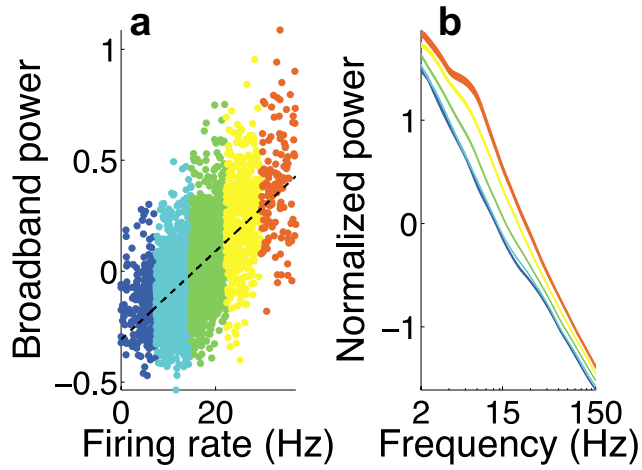

Figure 2. A representative neuron exhibiting a positive correlation between firing rate and broadband LFP power. $\boldsymbol{a}$, Broadband power and firing rate for the neuron analyzed in Figure 1. Each 500 ms epoch of the recording session is represented by one colored dot. The color of each dot represents its relative firing rate. Warm colors depict epochs with high firing rates, and cool colors indicate epochs with low firing rates. The dashed black line shows an ordinary leastsquares regression to these data. $\boldsymbol{b}$, Average LFP power spectra for epochs with different firing rates. The same color scheme is used in both panels. As firing rate increases, the power spectrum exhibits a positive shift at all observed frequencies. The thickness of each line represents $\pm 1 \mathrm{SEM}$

To determine whether this pattern was robust across the entire recording session, we examined the mean broadband LFP power and the mean firing rate for each of the 3477 half-second epochs we recorded for this neuron. Data from each epoch appear as a point in Figure $2 a$, where the horizontal coordinate indicates the firing rate and the vertical coordinate indicates the normalized broadband power. Across the entire recording session, these points were clustered along the diagonal, indicating that neuronal firing rate was positively correlated with LFP broadband power (Pearson's $r=0.6, p<10^{-10}$ ). Figure $2 b$ depicts this relation in a different manner, showing the mean LFP power spectra for each of five groups of epochs where this neuron had different firing rates (different colors in Fig. 2a). As this neuron's firing rate increased, the LFP power spectrum exhibited a proportional upward shift at all frequencies.

We next sought to identify all neurons in our dataset whose firing rates varied with broadband power (as in the example above) or with narrowband power (as documented in the previous literature). Because broadband power is influenced by each 
narrow frequency band, disambiguating broadband and narrowband effects is critical for understanding the relation between neuronal spiking and LFP activity. To identify neurons exhibiting each of these patterns, we fit a bivariate linear regression model to the relation between firing rate and measures of both broadband and narrowband LFP power. For each neuron, we computed the firing rate for each $500 \mathrm{~ms}$ epoch and we also computed LFP power measured at the same electrode at five narrow frequency bands: delta $(2-4 \mathrm{~Hz})$, theta $(4-8 \mathrm{~Hz})$, alpha $(8-12 \mathrm{~Hz})$, beta $(12-30 \mathrm{~Hz})$, and gamma $(30-150 \mathrm{~Hz})$, in addition to computing broadband power (see Materials and Methods). For each neuron, we then performed a set of bivariate regressions where broadband power and the mean power in one narrow frequency band were simultaneously used to predict the neuron's instantaneous firing rate. The two $\beta$ coefficients estimated in each regression indicate the contributions of broadband activity and this particular narrowband frequency band toward each neuron's firing rate (see Materials and Methods).

Combining the results of all five regressions for each neuron, we designated a neuron as a broadband-shift neuron when all five $\beta$ coefficients for the broadband predictor were significantly different from zero in the same direction. We designated a neuron as a narrowband-shift neuron if, across the five regressions, either (1) one and only one narrowband $\beta$ coefficient was significantly different from zero or (2) exactly two narrowband $\beta$ coefficients at adjacent frequency bands (e.g., beta and gamma) were significantly different from zero in the same direction. (An individual neuron could receive both the broadband-shift and narrowband-shift designations.) We adjusted our significance thresholds for each neuron to yield a 5\% falsepositive rate for each designation based on a reshuffling procedure (see Materials and Methods).

Figure 3 illustrates power spectra for neurons whose spiking (positively or negatively) correlated with narrowband LFP oscillations, neurons whose spiking correlated with broadband LFP activity, and neurons whose spiking was simultaneously influenced by both broadband and narrowband activity. Figure 3 also shows the proportion of neurons that fell into each category. For neurons whose firing rates were correlated with broadband or narrowband LFP patterns, the most prominent pattern in the dataset was that $34 \%$ of neurons exhibited spiking that was positively correlated with broadband LFP activity (Fig. 3, right column) - the prevalence of this phenomenon far exceeded the level expected by chance $\left(p<10^{-6}\right.$, binomial test). In addition to this broadband effect, $29.7 \%$ of all neurons varied their spiking in relation to narrowband LFP oscillations (top and bottom rows).

The broadband and narrowband patterns were qualitatively different: The broadband shift effect was remarkably unidirectional, with $92 \%$ of all broadband-shift neurons exhibiting this effect in a positive direction. In contrast, among narrowbandshift neurons, only $66 \%$ exhibited positive correlations. Both
Broadband shifts

N.s. Positive

Negative N.s.
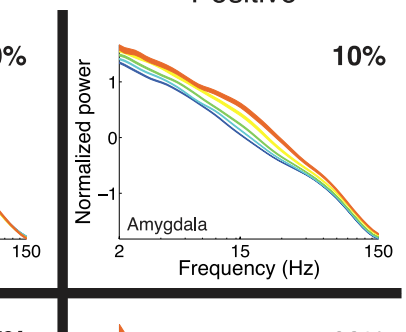

$10 \%$
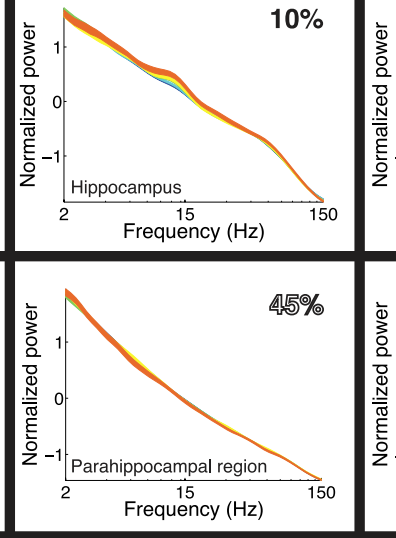

$23 \%$
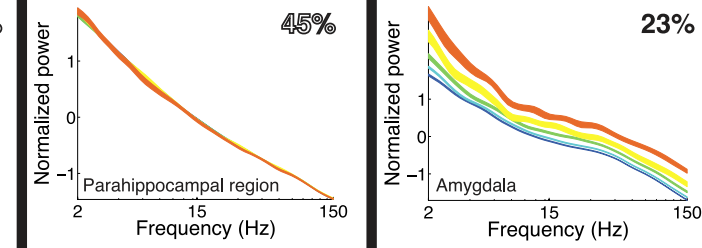

$8 \%$

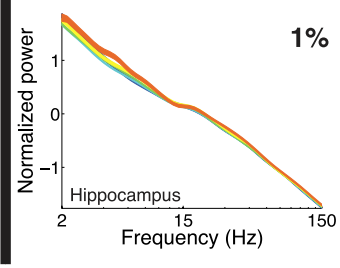

Frequency $(\mathrm{Hz})$

(a)

Figure 3. Categories of observed neurons. Each box corresponds to one of the nine possible categories into which our regression framework assigned each neuron in our dataset. The right and left columns correspond to significant positive and negative broadband shifts, respectively. The top and bottom rows correspond to significant positive and negative narrowband shifts, shifts, respectively. The proportion of neurons placed into each filled in black. Each box contains power spectra (in the same format as Fig. 2b) of an example neuron from the corresponding ach example neuron is indicated in the lower left corner of each box.

broadband and narrowband effects often appeared simultaneously at the same recording sites; we found that $11.7 \%$ of neurons were classified as both broadband- and narrowband-shift neurons (i.e., corner entries in Fig. 3). This proportion is greater than would be expected if broadband and narrowband phenomena were independent $\left(\chi_{(1)}^{2}=5.3, p<0.05\right)$.

We next sought to determine whether the phenomenon of positive broadband-shift neurons was linked to any particular brain regions. We found positive broadband-shift neurons in all observed brain regions (Fig. 4). To measure the prevalence of this pattern, we calculated the proportions of positive broadbandshift neurons in each of the five brain regions we examined (frontal and posterior cortices, parahippocampal region, amygdala, and hippocampus). In each of these regions, we observed significantly more positive broadband-shift neurons than expected by chance (binomial tests, $p<10^{-6}$ for each region). However, the prevalence of this effect significantly varied between regions $\left(\chi_{(4)}^{2}=34.01, p<10^{-6}\right)$. In particular, the proportion of neurons exhibiting significant positive broadband shift patterns was significantly greater in medial temporal regions than in other regions (Fig. 5) (post hoc $\chi_{(1)}^{2}=241.56, p<10^{-6}$ ).

The foregoing analyses focused on characterizing the broadband shift phenomenon and distinguishing it from narrowband effects. We next sought to determine whether combining our measure of broadband power with narrowband power can improve the prediction of firing rate. We performed a series of bivariate regressions with broadband power in conjunction with narrowband power, using a different narrow band (delta, theta, alpha, beta, or gamma) in each regression. Figure 6 shows that 

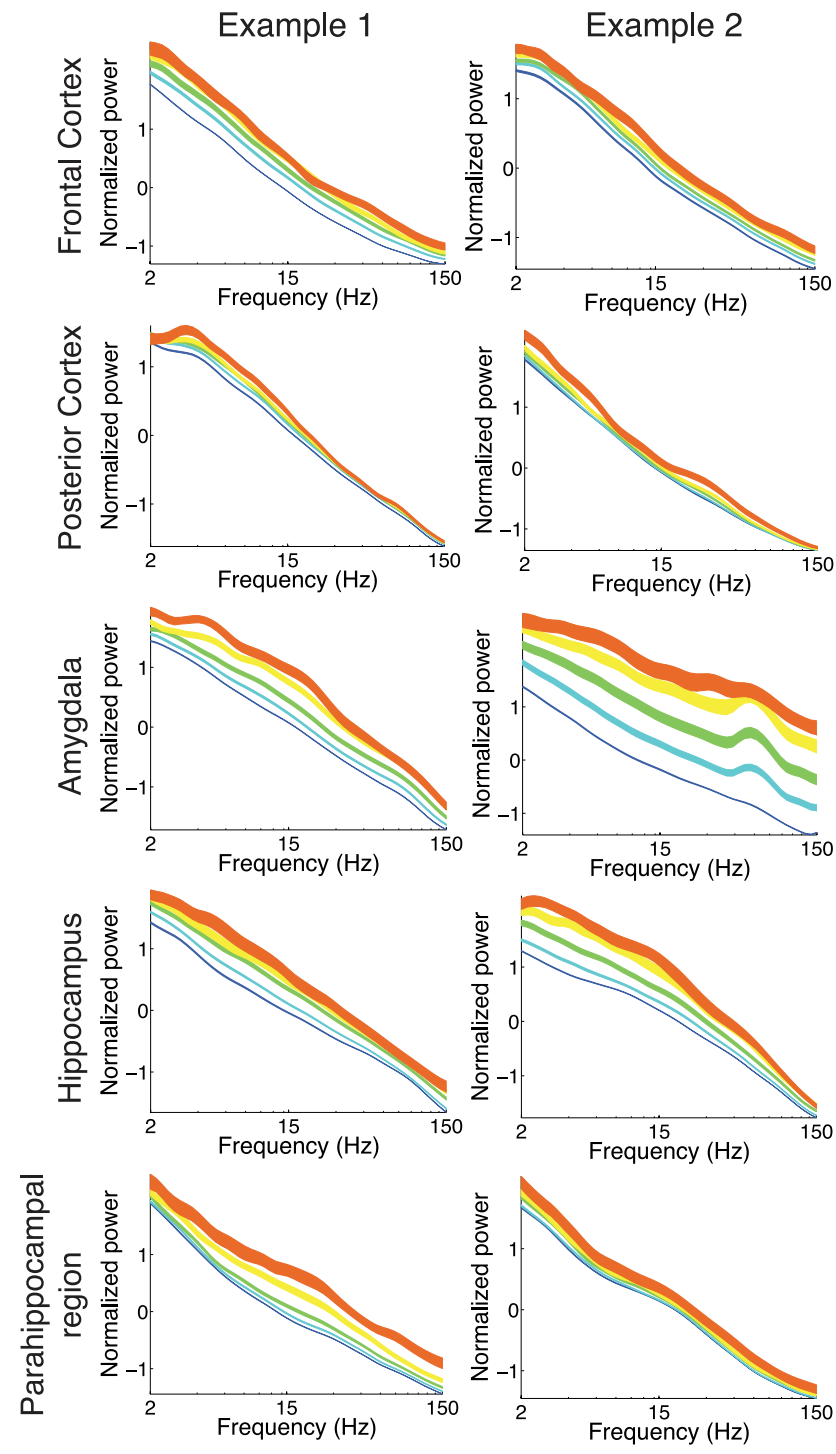

Figure 4. Broadband-shift neurons throughout the brain. Each row shows two example neurons from a single brain region. Within each row, each plot illustrates the power spectra from an example neuron, in the same format as Figure $2 b$. (Each of these neurons were categorized as positive broadband-shift neurons, corresponding to the middle-right box of Fig. 3.)

broadband and gamma-band power were the two dominant LFP measures that positively correlated with firing rate. The proportions of neurons exhibiting these two effects were comparable to one another and were both significantly greater than the proportions of significant positive or negative correlations observed at other frequency bands $\left(\chi_{(1)}^{2}>65\right.$ and $p<10^{-10}$ for all comparisons). In addition, we observed that in the delta, theta, and alpha bands, a greater number of neurons showed negative correlations with firing rate than positive correlations, which is consistent with recent literature (Mukamel et al., 2005). A notable feature of the broadband effects is that they are predominantly unidirectional, with nearly all (92\%) significant correlations being in the positive direction. In contrast, for neurons showing significant gamma-firing-rate correlations, only $63 \%$ showed positive correlations. Because the broadband effects were significantly more unidirectional than narrowband gamma effects $\left(\chi_{(1)}^{2}=171.4\right.$, $p<10^{-10}$ ), our results indicate that broadband power is a more specific predictor of increased neuronal spiking than narrowband oscillations.

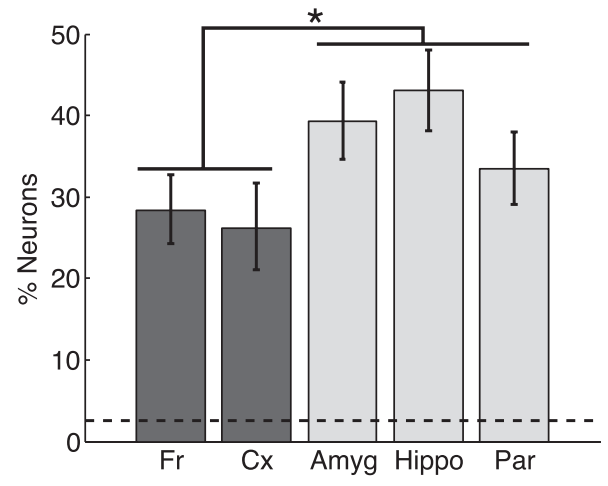

Figure 5. Percentage of positive broadband-shift neurons observed in each brain region. Dark gray bars correspond to neocortical regions: frontal cortex ( $\mathrm{Fr}$ ) and posterior cortex (Cx). Light gray bars correspond to medial temporal lobe structures: amygdala (Amyg), hippocampus (Hippo), and parahippocampal region (Par). Positive broadband-shift neurons were more concentrated in the medial temporal lobe than in the neocortex. The dotted horizontal black line marks the false-detection rate for positive broadband-shift neurons. Error bars indicate $95 \%$ confidence intervals based on the binomial distribution.

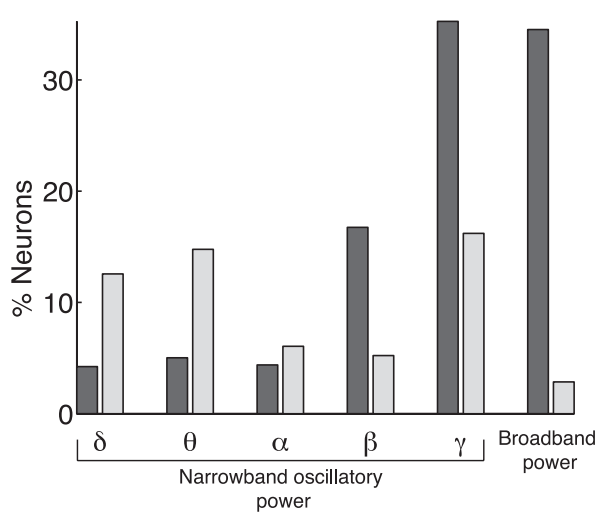

Figure 6. LFP components that predict firing rate. Dark gray bars indicate the percentage of neurons in each region that exhibited positive correlations between firing rate and a particular LFP feature; light gray bars show the percentage of neurons in each region that exhibited negative correlations. The bars on the left indicate the proportions of neurons whose firing rates were correlated with power in each narrow frequency band: delta $(2-4 \mathrm{~Hz})$, theta $(4-8 \mathrm{~Hz})$, alpha $(8-12 \mathrm{~Hz})$, beta $(12-30 \mathrm{~Hz})$, and gamma $(30-150 \mathrm{~Hz})$. Each neuron may be counted in at most one direction (i.e., either positive or negative) per narrow frequency band. The bars on the right indicate the proportions of neurons whose firing rates were correlated with broadband power (i.e., broadband-shift neurons).

To further illustrate the robust nature of the broadband phenomenon, we computed the average power spectra across all 2030 neurons in our dataset (Fig. 7a). Although only $37 \%$ of the neurons included in this figure demonstrated significant firing rate-related broadband shifts in their LFP power spectra (Fig. 7b), there is nevertheless a clear overall effect of increasing power at all frequency bands with increased neuronal firing. However, repeating this analysis after excluding all broadband-shift neurons (Fig. 3, left and right columns) reveals an effect of increased gamma power with increased firing rate (Fig. $7 c$ ). Thus, the population of non-broadband-shift neurons recovers the gammafiring-rate relation described in the prior literature (Mukamel et al., 2005).

\section{Discussion}

We examined the relation between the firing of individual neurons and simultaneous LFP activity. In previous work, LFP recordings have typically been analyzed in terms of narrowband oscillations, which indicate that nearby neurons are synchro- 
nously spiking at a particular frequency (Buzsáki, 2006; Fries et al., 2007; Jensen et al., 2007). For many of the neurons that we recorded, narrowband oscillations correlated significantly with firing rate, especially in the gamma frequency band. We also analyzed LFP recordings using a measure of broadband activity, which identifies fluctuations that simultaneously appear at all frequencies. This analysis revealed that increases in the power of broadband LFP activity positively correlated with the spiking of nearby neurons. (We verified that these broadband increases in LFP power were not the result of the appearance of spike waveforms in our recordings; see supplemental material, available at www.jneurosci.org.) We observed this broadband effect in multiple patients and brain regions, and found that it was most prevalent in medial temporal lobe structures. Our findings indicate that both narrowband and broadband components of the LFP can be used to estimate the firing rates of nearby neurons, even though LFP recordings do not actually display the waveforms of individual action potentials. Below we discuss these findings in relation to the recent literature on narrowband and broadband LFP phenomena.

\section{Narrowband effects}

A wide range of studies have examined the relation between narrowband LFP oscillations and the firing of nearby neurons, identifying an array of patterns that often differ across species, frequencies, brain regions, and behaviors. However, two common trends have emerged.

Generally in neocortex, the power of low-frequency (less than $\sim 10 \mathrm{~Hz}$ ) oscillations are negatively correlated with neuronal firing rates, while the power of high-frequency (more than $\sim 30 \mathrm{~Hz}$ ) oscillations are typically positively correlated with neuronal firing rates (Mukamel et al., 2005; Rasch et al., 2008). These results comport well with the observation of task-related increases in the amplitude of high-frequency activity and decreases in the amplitude of lower-frequency activity (Crone et al., 1998a,b; Miller et al., 2007b). In the present study, we recorded from distributed regions throughout the brains of neurosurgical patients and found narrowband effects that were consistent with this literature. In particular, we found that LFP oscillations at low frequencies (delta, theta, and alpha bands) were negatively correlated with neuronal spiking, while high-frequency LFP oscillations were most often positively correlated with neuronal spiking (beta and gamma bands). However, we also found a significant number of neurons whose firing rates were negatively correlated with gamma oscillations (Fig. 6). Thus, there is considerable variability in the relation between narrowband LFP oscillations and neuronal spiking across neurons.

\section{Broadband effects}

Our primary finding is that neuronal spiking is positively correlated with broadband LFP power. Broadband changes in LFP power are qualitatively different from narrowband changes: whereas narrowband power changes reflect modulations in oscillatory activity, broadband power changes generally reflect modulations in the variance of the LFP time series (Annibaldi and Hopcraft, 2002). Previous research has identified broadband power changes in brain regions that are thought to be involved with particular behaviors. One example is a study by Miller et al. (2007b), which showed that finger and tongue movements are accompanied by highly localized broadband LFP power increases in human motor cortex (also see Miller et al., 2008, 2009). These studies show that broadband LFP shifts can be difficult to identify in practice because they often occur simultaneously with power decreases at alpha and theta frequencies [e.g., see Miller et al. (2007b), their Figs. 1, 2]. Furthermore, oscillatory correlates of spatial and verbal memory processes are not always specific to a given frequency band, but rather appear at a very broad range of frequencies, consistent with a broadband effect (Ekstrom et al., 2007; Sederberg et al., 2007). Although few other studies have discussed their findings in terms of broadband patterns, a close inspection of the data from several studies reveals examples of behavior-related broadband LFP patterns. For example, Edwards et al. (2005) report that unexpected auditory stimuli were followed by LFP changes in leftfrontal and temporal cortex that appear to be broadband in nature [Edwards et al. (2005), their Fig. 3]. Furthermore, the data reported by Lachaux et al. (2005) are consistent with the presence of positive broadband power shifts in the fusiform gyrus during face viewing [Lachaux et al. (2005), their Fig. 2, top; also their Fig. 5, patient 3]. A third example can be found in Belitski et al.(2008), which examined LFP power spectra from macaque visual cortex during movie watching. Figure $2 a$ in their manuscript shows a positive broadband shift in the LFP power spectrum during movies, relative to a baseline period. Finally, Ball et al. (2008) show data suggesting that broadband patterns can be observed noninvasively using scalp electroencephalography (EEG). For example, they show recordings from an electrode above motor cortex that exhibits a broadband power increase (coupled with a low-frequency narrowband decrease) after movement [Ball et al. (2008), their Fig. 1a]. While each of these studies implies that broadband power indicates when a particular brain region is active, ours is the first to show that increases in broadband activity directly relate to simultaneous increases in neuronal spiking.

Given our finding that neuronal spiking is correlated with broadband power, one may wonder why many studies report task-related high-frequency (gamma) modulation rather than reporting broadband effects (Lachaux et al., 2005; Belitski et al., 2008; Ball et al., 2008). As described by Miller et al. (2007b), task-related spectral changes often appear as simultaneous lowfrequency decreases coupled with broadband increases in power. Due to these low-frequency decreases, in practice, broadband power increases are often most visible in the gamma band. Thus many of the gamma responses reported in the literature may actually be the result of broadband power increases coupled with low-frequency narrowband power decreases. 
An important feature of our methodology is the robust, nonlinear regression (Holland and Welsch, 1977) technique we used for distinguishing simultaneous changes in broadband and narrowband LFP activity. Because this regression is resistant to outliers, its estimate of broadband power is only minimally affected by the presence or absence of narrowband oscillations, which appear as narrowband peaks in the power spectrum. In contrast, linear methods might interpret the appearance of large narrowband oscillations as increased broadband power. This issue can be important, because broadband and narrowband changes often occur simultaneously and in opposite directions (Miller et al., 2007b, 2009; Ball et al., 2008), which can make these effects difficult to tease apart.

\section{Broader implications}

A particularly exciting implication of this study is that increases (Miller et al., 2007b) and decreases (Lachaux et al., 2008) in broadband power, which can be observed from both invasive (Edwards et al., 2005; Lachaux et al., 2005; Ekstrom et al., 2007; Miller et al., 2007b, 2009; Sederberg et al., 2007) and noninvasive (Ball et al., 2008) recordings, may be used as a proxy for neuronal spiking. This is especially important for researchers using macroelectrode recordings to study the neural basis of human behavior, because such recordings cannot resolve single-neuron spiking, but can record these broadband shifts. To the extent that the broadband effects observed here generalize to activity recorded at broader spatial scales, one could use broadband activity to measure correlates of neuronal spiking in scalp recorded EEG and MEG signals, or even to modulate neuronal spiking using realtime feedback methods (Lachaux et al., 2007; Miller et al., 2007a). In this way, the broadband power measure proposed here may lead to new discoveries concerning how neuronal activity underlies complex human behavior and cognition.

\section{References}

Annibaldi SV, Hopcraft KI (2002) Random walks with power-law fluctuations in the number of steps. J Phys A Math Gen 35:8635-8645.

Ball T, Demandt E, Mutschler I, Neitzel E, Mehring C, Vogt K, Aertsen A, Schulze-Bonhage A (2008) Movement related activity in the high gamma range of the human EEG. Neuroimage 41:302-310.

Belitski A, Gretton A, Magri C, Murayama Y, Montemurro MA, Logothetis NK, Panzeri S (2008) Low-frequency local field potentials and spikes in primary visual cortex convey independent visual information. J Neurosci 28:5696-5709.

Buzsáki G (2004) Large-scale recording of neuronal ensembles. Nat Neurosci 7:446-451.

Buzsáki G (2006) Rhythms of the brain. New York: Oxford UP.

Canolty RT, Edwards E, Dalal SS, Soltani M, Nagarajan SS, Kirsch HE, Berger MS, Barbaro NM, Knight RT (2006) High gamma power is phaselocked to theta oscillations in human neocortex. Science 313:1626-1628.

Caplan JB, Madsen JR, Schulze-Bonhage A, Aschenbrenner-Scheibe R, Newman EL, Kahana MJ (2003) Human theta oscillations related to sensorimotor integration and spatial learning. J Neurosci 23:4726-4736.

Chen CM, Lakatos P, Shah AS, Mehta AD, Givre SJ, Javitt DC, Schroeder CE (2007) Functional anatomy and interaction of fast and slow visual pathways in macaque monkeys. Cereb Cortex 17:1561-1569.

Crone NE, Miglioretti DL, Gordon B, Sieracki JM, Wilson MT, Uematsu S, Lesser RP (1998a) Functional mapping of human sensorimotor cortex with electrocorticographic spectral analysis. I. Alpha and beta eventrelated desynchronization. Brain 121:2271-2299.

Crone NE, Miglioretti DL, Gordon B, Lesser RP (1998b) Functional mapping of human sensorimotor cortex with electrocorticographic spectral analysis. II. Event-related synchronization in the gamma band. Brain 121:2301-2315.

Crone NE, Sinai A, Korzeniewska A (2006) High-frequency gamma oscillations and human brain mapping with electrocorticography. Prog Brain Res 159:275-295.
Cruikshank SJ, Rose HJ, Metherate R (2002) Auditory thalamocortical synaptic transmission in vitro. J Neurophysiol 87:361-384.

Edwards E, Soltani M, Deouell LY, Berger MS, Knight RT (2005) High gamma activity in response to deviant auditory stimuli recorded directly from human cortex. J Neurophysiol 94:4269-4280.

Ekstrom AD, Caplan JB, Ho E, Shattuck K, Fried I, Kahana MJ (2005) Human hippocampal theta activity during virtual navigation. Hippocampus 15:881-889.

Ekstrom A, Viskontas I, Kahana M, Jacobs J, Upchurch K, Bookheimer S, Fried I (2007) Contrasting roles of neural firing rate and local field potentials in human memory. Hippocampus 17:606-617.

Fried I, Wilson CL, Maidment NT, Engel J Jr, Behnke E, Fields TA, MacDonald KA, Morrow JW, Ackerson L (1999) Cerebral microdialysis combined with single-neuron and electroencephalographic recording in neurosurgical patients. J Neurosurg 91:697-705.

Fries P, Reynolds JH, Rorie AE, Desimone R (2001) Modulation of oscillatory neuronal synchronization by selective visual attention. Science 291:1560-1563.

Fries P, Nikolić D, Singer W (2007) The gamma cycle. Trends Neurosci 30:309-316.

Henrie JA, Shapley R (2005) LFP power spectra in V1 cortex: the graded effect of stimulus contrast. J Neurophysiol 94:479-490.

Holland PW, Welsch RE (1977) Robust regression using iteratively reweighted least-squares. Commun Stat Theory Methods A 6:813-827.

Jacobs J, Kahana MJ (2009) Neural representations of individual stimuli in humans revealed by gamma-band ECoG activity. J Neurosci 29:10203-10214.

Jacobs J, Kahana MJ, Ekstrom AD, Fried I (2007) Brain oscillations control timing of single-neuron activity in humans. J Neurosci 27:3839-3844.

Jensen O, Kaiser J, Lachaux JP (2007) Human gamma-frequency oscillations associated with attention and memory. Trends Neurosci 30:317-324.

Jerbi K, Ossandón T, Hamamé CM, Senova S, Dalal SS, Jung J, Minotti L, Bertrand O, Berthoz A, Kahane P, Lachaux JP (2009) Task-related gamma-band dynamics from an intracerebral perspective: review and implications for surface EEG and MEG. Hum Brain Mapp 30:1758-1771.

Juergens E, Guettler A, Eckhorn R (1999) Visual stimulation elicits locked and induced gamma oscillations in monkey intracortical- and EEGpotentials, but not in human EEG. Exp Brain Res 129:247-259.

Kahana MJ (2006) The cognitive correlates of human brain oscillations. J Neurosci 26:1669-1672.

Kahana MJ, Seelig D, Madsen JR (2001) Theta returns. Curr Opin Neurobiol 11:739-744.

Kanisza G (1979) The organization of vision. New York: Praeger.

Kaur S, Lazar R, Metherate R (2004) Intracortical pathways determine breadth of subthreshold frequency receptive fields in primary auditory cortex. J Neurophysiol 91:2551-2567.

Koffka K (1935) Principles of gestalt psychology. New York: Harcourt, Brace and World.

Köhler W (1947) Gestalt psychology. New York: Liveright.

Kreiman G (2007) Brain science: from the very small to the very large. Curr Biol 17:R768-R770.

Kreiman G, Hung CP, Kraskov A, Quiroga RQ, Poggio T, DiCarlo JJ (2006) Object selectivity of local field potentials and spikes in the macaque inferior temporal cortex. Neuron 49:433-445.

Lachaux JP, George N, Tallon-Baudry C, Martinerie J, Hugueville L, Minotti L, Kahane P, Renault B (2005) The many faces of the gamma band response to complex visual stimuli. Neuroimage 25:491-501.

Lachaux JP, Jerbi K, Bertrand O, Minotti L, Hoffmann D, Schoendorff B, Kahane P (2007) A blueprint for real-time functional mapping via human intracranial recordings. PLoS One 2:e1094.

Lachaux JP, Jung J, Mainy N, Dreher JC, Bertrand O, Baciu M, Minotti L, Hoffmann D, Kahane P (2008) Silence is golden: transient neural deactivation in the prefrontal cortex during attentive reading. Cereb Cortex 18:443-450.

Logothetis NK (2003) The underpinnings of the BOLD functional magnetic resonance imaging signal. J Neurosci 23:3963-3971.

Miller KJ, den Nijs M, Shenoy P, Miller JW, Rao RP, Ojemann JG (2007a) Real-time functional brain mapping using electrocorticography. Neuroimage 37:504-507.

Miller KJ, Leuthardt EC, Schalk G, Rao RPN, Anderson NR, Moran DW, 
Miller JW, Ojemann JG (2007b) Spectral changes in cortical surface potentials during motor movement. J Neurosci 27:2424-2432.

Miller KJ, Shenoy P, den Nijs M, Sorensen LB, Rao RPN, Ojemann JG (2008) Beyond the gamma band: the role of high-frequency features in movement classification. IEEE Trans Biomed Eng 55:1634-1637.

Miller KJ, Zanos S, Fetz EE, den Nijs M, Ojemann JG (2009) Decoupling the cortical power spectrum reveals real-time representation of individual finger movements in humans. J Neurosci 29:3132-3137.

Milstein J, Mormann F, Fried I, Koch C (2009) Neuronal shot noise and Brownian $1 / f^{2}$ behavior in the local field potential. PLoS One 4:e4338.

Mitzdorf U (1985) Current source-density method and application in cat cerebral cortex: investigation of evoked potentials and EEG phenomena. Physiol Rev 65:37-100.

Mukamel R, Gelbard H, Arieli A, Hasson U, Fried I, Malach R (2005) Coupling between neuronal firing, field potentials, and fMRI in human auditory cortex. Science 309:951-954.

Newman EL, Caplan JB, Kirschen MP, Korolev IO, Sekuler R, Kahana MJ (2007) Learning your way around town: how virtual taxicab drivers learn to use both layout and landmark information. Cognition 104:231-253.

Pal NR, Pal SK (1993) A review of image segmentation techniques. Pattern Recognit Lett 26:1277-1294.

Percival DB, Walden AT (1993) Spectral analysis for physical applications. Cambridge, UK: Cambridge UP.
Pesaran B, Pezaris JS, Sahani M, Mitra PP, Andersen RA (2002) Temporal structure in neuronal activity during working memory in macaque parietal cortex. Nat Neurosci 5:805-811.

Quiroga RQ, Nadasdy Z, Ben-Shaul Y (2004) Unsupervised spike detection and sorting with wavelets and superparamagnetic clustering. Neural Comput 16:1661-1687.

Rasch MJ, Gretton A, Murayama Y, Maass W, Logothetis NK (2008) Inferring spike trains from local field potentials. J Neurophysiol 99:1461-1476.

Sederberg PB, Schulze-Bonhage A, Madsen JR, Bromfield EB, McCarthy DC, Brandt A, Tully MS, Kahana MJ (2007) Hippocampal and neocortical gamma oscillations predict memory formation in humans. Cereb Cortex 17:1190-1196.

Singer W, Gray CM (1995) Visual feature integration and the temporal correlation hypothesis. Annu Rev Neurosci 18:555-586.

Tanji K, Suzuki K, Delorme A, Shamoto H, Nakasato N (2005) Highfrequency $\gamma$-band activity in the basal temporal cortex during picturenaming and lexical-decision tasks. J Neurosci 25:3287-3293.

von der Malsburg C (1981) The correlation theory of brain function. Internal Report 81-2. Göttingen, Germany: Max-Planck-Institute for Biophysical Chemistry.

Witter M (2002) The parahippocampal region: past, present and future. In: The parahippocampal region: organization and role in cognitive functions (Witter M, Wouterlood F, eds), pp 3-19. Oxford, UK: Oxford UP. 